\title{
Frustration of Bragg reflection by cooperative dual-mode interference: a new mode of optical propagation
}

\author{
Amnon Yariv \\ California Institute of Technology, Pasadena, California 91125-0001
}

Received July 20, 1998

\begin{abstract}
A new optical mode of propagation is described, which is the natural eigenmode (supermode) of a fiber (or any optical waveguide) with two cospatial periodic gratings. The mode frustrates the backward Bragg scattering from the grating by destructive interference of its two constituent submodes (which are eigenmodes of a uniform waveguide). It can be used in a new type of spatial mode conversion in optical guides. (c) 1998 Optical Society of America
\end{abstract}

OCIS codes: $\quad 050.2770,060.2310,030.4070$.

Consider an optical mode $|a\rangle$ propagating in a fiber that is incident upon a grating with a period $\Lambda_{1}$ and satisfies the Bragg condition

$$
\beta_{a}+\beta_{b}=\frac{2 \pi}{\Lambda_{1}}
$$

which is necessary for strong coherent scattering from right-traveling mode $|a\rangle$ into left-traveling mode $|b\rangle$. $\beta_{a}$ and $-\beta_{b}$ are the propagation constants of the two guided modes. This situation is depicted in Fig. 1(a). Also shown is a momentum diagram in which the values of $\beta$ (momenta) of the various uniform fiber modes involved in the scattering as well as the grating momentum are shown. The scattering causes the intensity of mode $\mid a>$ to decay evanescently in the grating region of the waveguide. Typical fiber gratings can yield an $|a>\rightarrow| b>$ reflection that exceeds $99 \%$ in less than $1 \mathrm{~cm}$ of grating length. ${ }^{1}$

A similar fate awaits a higher transverse mode, $|c\rangle$, propagating in the fiber when the mode is incident upon a second grating, 2 , with a period $\Lambda_{2}$ that satisfies

$$
\beta_{b}+\beta_{c}=\frac{2 \pi}{\Lambda_{2}}
$$

This mode will also undergo scattering into the same backward mode, $\mid b>$, as shown in Fig. 1(b).

Now consider what happens when gratings 1 and 2 are cospatial, i.e., occupy the same $z$ stretch of the fiber. If the forward-propagating fields of $|a\rangle$ and $|c\rangle$ are temporally coherent, then the total scattering into $|b\rangle$ is obtained by an algebraic addition of the $|a\rangle \rightarrow|b\rangle$ and the $|c>\rightarrow| b>$ scattering amplitudes, as shown in Fig. 1(c). There exists, obviously, a ratio of the modal amplitudes $A / C$ of the two forward modes for which the net backward scattering $S_{a b}+S_{c b}$ into $\mid b>$ is zero. Under these conditions the backward mode $|b\rangle$, if it is not present at $z=0$, the beginning of the dual-grating region, will not be excited. Modes $\mid a>$ and $|c\rangle$ under this condition lose no power, thus defeating the Bragg scattering. It follows, self-consistently, that the above superposition of uniform fiber modes $|a\rangle$ and $|c\rangle$ constitutes a supermode of the dual-grating fiber. This new mode of optical propagation propagates

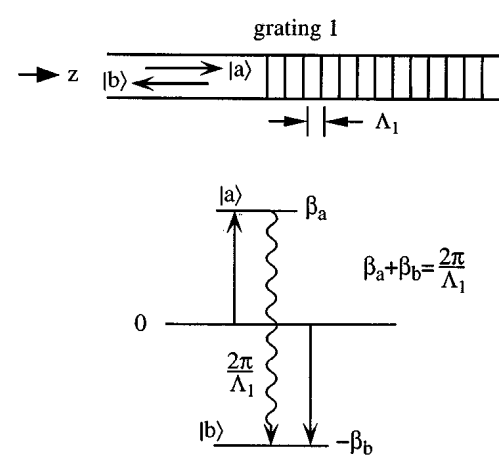

(a)
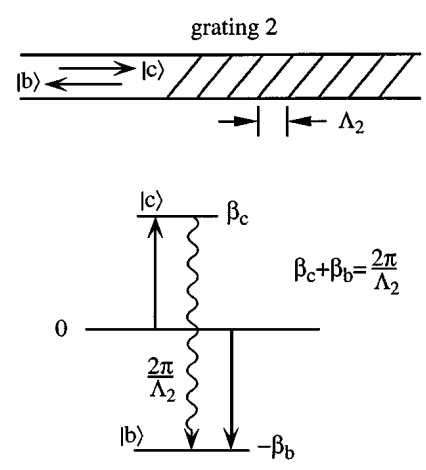

(b)
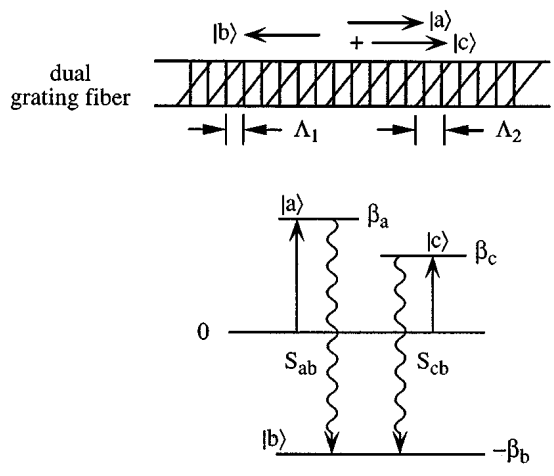

(c)

Fig. 1. (a) A fiber (or optical waveguide) modulated spatially by a grating with a period $\Lambda_{1}$, which Bragg scatters resonantly between forward mode $\mid a>$ and backward mode $\mid b>$. A momentum diagram shows the initial and the final photon momenta, $\beta$, involved as well as the lattice momentum $2 \pi / \Lambda_{1}$. (b) Fiber with a second grating $\left(\Lambda_{2}\right)$, which Bragg scatters a higher-order forward mode $\mid c>$ into backward mode $\mid b>$. (c) Dual-grating fiber, which can scatter resonantly and simultaneously between modes $\mid a>$ and $\mid c>$ and backward mode $\mid b>$ but not between modes $\mid a>$ and $\mid c>$. 
without loss through the dual-grating region that is essentially impenetrable by either $\mid a>$ or $\mid c>$ alone.

To formalize the above description we consider the coupled-mode equations that describe the interaction among the uniform fiber modes $|a\rangle,|b\rangle$, and $|c\rangle$, all different, that is due to the dual grating as in Fig. 1(c). The complex normalized modal amplitudes are $A, B$, and $C$ such that the total field at $z$ is given by

$$
\begin{aligned}
E(x, y, z)= & A(z) E_{1}(x, y) \exp \left(-i \beta_{a} z\right)+B(z) E_{3}(x, y) \\
& \times \exp \left(i \beta_{b} z\right)+C(z) E_{2}(x, y) \exp \left(-i \beta_{c} z\right) .
\end{aligned}
$$

Representing grating 1 by its coupling constant $K_{1}$ and grating 2 by $K_{2}$, we can describe the interaction by the coupled-mode equations ${ }^{2}$

$$
\begin{aligned}
\frac{\mathrm{d} A}{\mathrm{~d} z}= & i K_{1} B \exp \left[i\left(\beta_{a}+\beta_{b}-G_{1}\right) z\right] \\
\frac{\mathrm{d} B}{\mathrm{~d} z}= & -i K_{1} A \exp \left[-i\left(\beta_{a}+\beta_{b}-G_{1}\right) z\right]-i K_{2} C \\
& \times \exp \left[-i\left(\beta_{b}+\beta_{c}-G_{2}\right) z\right] \\
\frac{\mathrm{d} C}{\mathrm{~d} z}= & i K_{2} B \exp \left[i\left(\beta_{c}+\beta_{b}-G_{2}\right) z\right] \\
G_{1,2} \equiv & \frac{2 \pi}{\Lambda_{1,2}}
\end{aligned}
$$

Modes $\mid a>$ and $|c\rangle$ are not directly coupled. For such coupling to occur we require a grating with a long period $\Lambda$, where $\Lambda=2 \pi /\left(\beta_{a}-\beta_{c}\right)$. There exists, however, indirect coupling between $|a\rangle$ and $|c\rangle$, which is mediated by $\mid b>$ and is expressed by Eqs. (4)-(6). Nonlinear interactions in the writing of the grating, which could lead to grating momenta $m\left(2 \pi / \Lambda_{1}\right) \pm$ $n\left(2 \pi / \Lambda_{2}\right), m$ and $n$ integers, are assumed negligible.

From Eq. (5) it follows that when

$$
\begin{aligned}
\frac{A}{C} & =-\frac{K_{2}}{K_{1}}, \\
\Delta & \equiv\left(\beta_{c}-\beta_{a}+G_{1}-G_{2}\right)=0, \\
\frac{\mathrm{d} B}{\mathrm{~d} z} & =0 .
\end{aligned}
$$

If $B(0)=0$, then $B(z)=0$ and $A(z)$ and $C(z)$ are constant; i.e., there is no loss of forward-propagating power. We can obtain the supermode, i.e., the stable ratio of $A, B$, and $C$ in Eq. (3), by diagonalizing interaction equations (4)-(6). The solution that corresponds to our mode can be represented symbolically by a column vector (unnormalized):

$$
\tilde{E}_{1}=\left\{\begin{array}{c}
\frac{-K_{2}}{K_{1}} \exp \left[-i\left(G_{1}-G_{2}\right) z\right] \\
0 \\
\exp \left[-\frac{i}{2}\left(G_{2}-G_{1}\right) z\right]
\end{array}\right\} \operatorname{exp[-i(\beta _{a}+\beta _{c})z]}
$$

where we have chosen both individual Bragg mismatches as zero; i.e., $\beta_{a}+\beta_{b}=G_{1}$ and $\beta_{b}+\beta_{c}=G_{2}$. The remaining two solutions involve $B \neq 0$ and are not of interest in the present context.

Strictly speaking, this special mode in which the reflected component is dark $(B=0)$ exists only when resonance condition (8) is satisfied. The consequences of deviations from Eq. (8) resonance are discussed separately.

The existence of this new mode of propagation leads to some interesting applications and phenomena. If we allow $K_{1}$ and $K_{2}$ to depend on $z$ adiabatically, ${ }^{3,4}$ then according to Eq. (9) we can cause $\tilde{E}_{1}$ to vary between

$$
\left(\begin{array}{l}
1 \\
0 \\
0
\end{array}\right), \quad \text { when } \frac{K_{1}(z)}{K_{2}(z)}=0
$$

and

$$
\left(\begin{array}{l}
0 \\
0 \\
1
\end{array}\right), \quad \text { when } \frac{K_{2}(z)}{K_{1}(z)}=0
$$

i.e., we can bring about $|a>\leftrightarrow| c>$ mode conversion by mere adiabatic control of the gratings' profile. Note that mode $|b\rangle$ is never excited. This mode conversion is expected to have the high wavelength selectivity that is characteristic of backward Bragg scattering; i.e., $\Delta \lambda / \lambda \approx \lambda / 2 n L$, where $L$ is the grating length. This new form of mode conversion by adiabatic grating evolution is the spatial equivalent of coherent temporal population control in atomic physics. ${ }^{5}$ This subject will be considered in detail in the future.

The author is indebted to Phil Willems and to Eva Peral for many stimulating discussions. This research was supported by the Defense Advanced Research Projects Agency, the U.S. Office of Naval Research, and the U.S. Air Force Office of Scientific Research. This support is gratefully acknowledged.

\section{References}

1. T. Erdogan, J. Lightwave Technol. 15, 8 (1997).

2. A. Yariv, IEEE J. Quantum Electron. QE-9, 919 (1973).

3. D. Marcuse, Theory of Dielectric Optical Waveguides (Academic, New York, 1974), p. 106.

4. A. W. Snyder and J. D. Love, Optical Waveguide Theory (Chapman \& Hall, London, 1983).

5. M. P. Fewel, B. W. Shore, and K. Bergmann, Aust. J. Phys. 50, 281 (1977). 